\title{
Ludwig Krämer Die Europäische Union und der Export von Abfällen in die Dritte Welt
}

Der Export von Abfällen in andere Länder hat Konjunktur. Für die Bundesrepublik billiger als die Entsorgung im eigenen Land, für Drittländer eine lukrative Einnahmequelle, hat es die Kontrolle des Abfallexports schwer, wirksam zu werden. Der Autor zeichnet die einzelnen europäischen Regelungen nach, die verhindern sollen, daß der Export von Abfall zu einem umweltgefäbrdenden Geschäft wird oder ein solches bleibt.

Die Red.

Jedes Produkt wird früher oder später zu Abfall. Die Europäische Union, zu deren Zielen die Schaffung und Vollendung eines Binnenmarktes für Produkte gehört, bei dem Staatsgrenzen keine Wirtschaftsgrenzen mehr sind, hat sich daher schon frühzeitig, nämlich seit 1975, mit Problemen der Abfallbewirtschaftung beschäfrigt: die ersten Richtlinien' in Bereich des Umweltschutzes betrafen Abfallfragen². Das Aufkommen von gesetzlichen Vorschriften zur Abfallbewirtschaftung - Sammeln, Befördern, Verwerten, Verbrennen, Ablagern - in den sechziger und siebziger Jahren in allen Staaten Westeuropas und auf Gemeinschaftsebene konzentrierte sich zunächst auf den Blick nach innen, $d$.h. auf das Anpacken der Probleme im eigenen Land. Der Export von Abfällen insbesondere in Länder der Dritten Welt fand erst später Aufmerksamkeit.

Der folgende Beitrag zeichnct die Entwicklung der Abfallexporte aus den Industrieländern, insbesonderc der Europäischen Gemeinschaft in den letzten fünfundzwanzig Jahren nach. Er konzentriert sich dabei auf die Ebene der Europäischen Union (Europäischen Gemeinschaft - EG) und, soweit erforderlich, die internationale Ebene. Einzelstaatliche Enrwicklungen werden nicht erörtert.

\section{Abfallregelungen der $E G$}

Die EG-Richtlinie über Abfälle von $1975^{3}$ stellte allgemeine Regeln für Abfälle und ihre Beseitigung auf; unter Bescitigung verstand sic - eine Definition, die in späteren Jahren wichrig wurde -:

»- das Einsammeln, Sortieren, Befördern und Behandeln von Abfällen und deren Lagerung und Ablagerung auf dem Boden oder im Boden;

- die erforderlichen Umwandlungsvorgänge zu ihrer Wiederverwendung, Rückgewinnung oder Verwertung".

1 Richtlinien nach EG-Recht sind verbindliche Regelungen, die dic Mitgliedstaaten zum Erreichen eines bescimmten Zicles verpflichten, ihnen aber die Wahl der Form und Mittel zur Erreichung dieses Zieles überlassen; inhaltlich stehen sie also gesetzlichen Regelungen viel näher als Empfehlungen.

2 Richclinie 75/439 vom 16.6.1975 über die Altölbeseitigung, AB|.EG 1975, Nr. L 195, S. 31; Richrlinie 75/ $44^{2}$ vom is-7. 1975 viber Abfälle, ABl.EG 1975, Nr. L 194, S. 47.

3 Richtlinie $75 / 442\left(F_{n} 2\right)$. 
Die Mitgliedstaaten hatten Maßnahmen zu treffen, um sicherzustellen, daß Abfälle beseitigt wurden, ohne die menschliche Gesundheit zu gefährden oder die Umwelt zu schädigen (Artikel 4). Irgendeine geographische Beschränkung, etwa auf das Hoheitsgebiet der Mitgliedstaaten, war in diesen Vorschriften nicht enthalten. An sich galten sie daher auch dann, wenn Abfälle aus der Gemeinschaft heraus exportiert wurden. Soweit ersichtlich hat indessen kein Mitgliedstaat die Richtlinie auf Abfallexporte aus der Gemeinschaft heraus angewandt; es gab auch keine Versuche der Europäischen Kommission, eine solche Anwendung durchzusetzen.

1976 schlug die Kommission eine Richtlinie vor, um das Einbringen von Abfällen ins Meer zu verbieten'. Die EG-Mitgliedstaaten zogen indessen eine Regelung des Einbringens von Abfällen ins Meer im Wege internationaler Übereinkommen vor. Der Grund hierfür dürfte in der größeren nationalen Freiheit beim Aushandeln und insbesondere beim Durchführen internationaler Übereinkommen liegen. Jedenfalls wurde der Vorschlag der Kommission - wie auch ähnliche Vorschläge von 1985 und $1988^{6}$-im Rat nicht diskutiert, geschweige denn angenommen. Bis heute gelten daher für das Einbringen von Abfällen ins Meer neben den gemeinschaftlichen Rahmenvorschriften die Vorschriften der Übereinkommen von Paris, Helsinki und Barcelona, die auch von der Gemeinschaft ratifiziert sind, deren Einhaltung die Kommission indessen nicht überwacht.

1978 nahm der Rat eine Richtlinie für gefährliche Abfälle an ${ }^{7}$. Die Richtlinie definierte "giftige und gefährliche" Abfälle und verlangte erneut das Treffen von Maßnahmen, um eine Beseitigung der gefährlichen Abfälle ohne Gefährdung von Mensch und Umwelt sicherzustellen. Auch sie enthielt keine Bestimmungen zur Frage des Exports von Abfällen aus der Gemeinschaft. Auch sie wurde de facto auf Abfallexporte nicht angewandt.

Erst zu Beginn der achtziger Jahre kam es zu Regelungen über das Verbringen von Abfällen. Anfang 1983 legte die Kommission den Vorschlag für eine Richtlinie über den Transport gefährlicher Abfälle vor ${ }^{8}$, den sie unter dem Eindruck des Skandals der Dioxin-Fässer von Seveso 9 wesentlich verschärfte. Der Rat nahm. Ende 1984 die entsprechende Richtlinie an ${ }^{10}$.

Die Richtlinie führte für das Verbringen gefährlicher Abfälle ein Notifizierungsverfahren ein: der Besitzer von Abfällen, der sie in einen anderen Staat verbringen wollte, hatte dies den Behörden des Staates, in den die Abfälle verbracht werden sollte, anzuzeigen und bestimmte Angaben über Art und Menge der Abfälle und die Art ihrer Beseitigung zu machen; die Verbringung konnte erst beginnen, wenn der Bestimmungsstaat eine Empfangsbestätigung erteilte, was ihm die Möglichkeit gab, Einwände gegen die Verbringung zu erheben.

Bei Exporten in einen nicht zur EG gehörigen Staat mußte der Besitzer der Abfälle die Zustimmung des Bestimmungsstaates erhalten, bevor er das Notifizierungsverfahren einleitete. Indessen enthielten die Bestimmungen der Richtlinie - von den Fällen des

\footnotetext{
4 Die Europäischc Kommission hat nach Arrikel 1ss EG-Vcrrag die Aufgabe, -für die Anwendung diese Verrages suivic der von den Organen der Gemeinschafe getroffenen Bestimmungen Sorge zu tragen*. 5 ABl.EG 1976, Nr. C 40, S. 3 .

6 ABI.EG 1985 , Nr. C 245, S. 23 und KOM (88)8 yorn 7. 1. 1988.

7 Richtlinic $78 / 319$, ABl.EG 1978 , Nr. L 84, S. 43

8 ABI.EG 1983 , Nr. C $53, \mathrm{~S}_{3}$

9 Bei diesem Skandal waren 41 mit dioxin-halrigen Abfällen gefullte Fässer, die aus einem schweren Industrieunfall in Seveso stammten, 1982/83 heimlich und unter Verstoß gegen geltende Vorschriften aus Italien herausgebracht worden und dann von dem franzosischen Transportunternchmer in Frankrcich versteckt worden. Zahlreiche Staaten in Westeuropa suchten wochenlang nach den Fässern.

10 Richtinie $84 / 631$ uber die Ujberwachung und Kontrolle - in der Gemeinschaft - der grenzüberschreitenden Verbringung gefährlicher Abfälle, ABl.EG r 984, Nr. L 326, S. 31 ; die Richtlinie wurde 1985, 1986 und 1987 geändert; zitiert wird im folgenden nach dem geanderten Text.
} 
Abfalltransits durch die EG abgesehen - überhaupt keine Bestimung darüber, wer im

Falle eines Exports in einen nicht zur EG gehörenden Staat die Empfangsbestätigung erteilte und gegebenenfalls Einwendungen gegen die Verbringung geltcndmachen konnte, etwa, weil die Zustimmung des Bestimmungsstaates nicht vorlag.

Die Mitgliedstaaten der EG hatten die Richtlinie in innerstaatliches Recht umzusetzen, ihre Einhaltung sicherzustellen und der Kommission alle zwei Jahre über die Durchführung der Richtlinie zu berichten; die Kommission hatte einen EG-Bericht zu erstellen. Nicht ein einziger derartiger EG-Bericht wurde jemals erstellt.

\section{Das Baseler Übereinkommen über gefährliche Abfälle und die Reaktion der Industrieländer}

\section{Das Baseler Übereinkommen}

Ende der achtziger Jahre kam es unter der Ägide der Vereinten Nationen zu Verhandlungen über ein internationales Übereinkommen für Abfalltransporte, die schließlich am 22.3.1989 zum Abschluß des Baseler Übereinkommens über die Kontrolle der grenzüberschrcitenden Verbringung gefährlicher Abfälle und ihrer Entsorgung führten. Das Übereinkommen, dem die EG 1993 bcitrat ${ }^{11}$ und dem inzwischen etwa $x 20$ Staaten, darunter alle EG-Mitgliedstaaten beigetreten $\operatorname{sind}^{12}$, stellt den Grundsatz auf, daß gefährliche Abfälle in dem Staat zu beseitigen sind, in dem sie entstehen, wobei erneut unter »beseitigen " sowohl die endgültige Beseitigung als auch die Wiederverwertung von Abfällen verstanden wird. Das von dem Übereinkommen geschaffene System beruht auf dem »prior informed consent«-Grundsatz: der exportierende Staat muß den Bestimmungsstaat von der beabsichtigten Verbringung von gefährlichem Abfall unterrichten, und zwar schriftlich und in einer für den Bestimmungsstaat akzeptablen Sprache. Der Bestimmungsstaat muß dann der Verbringung zustimmen. Der exportierende Staat muß sich außerdem vergewissern, daß zwischen dem Exporteur und dem Empfänger der gefährlichen Abfälle ein Vertrag besteht, der eine umweltverträgliche Beseitigung der Abfälle festlegt. Jeder Verbringung von Abfällen sind entsprechende Begleitdokumente beizufügen, die über Herkunft, Art und Zusammensetzung, Bestimmung usw. der Abfälle Auskunft geben.

Auf Drängen insbesondere der Staaten der Dritten Welt beschloß die Zweite Konferenz der Vertragsstaaten des Baseler Übereinkommens am 25.3. 1994 einstimmig also mit den Stimmen aller EG Mitgliedstaaten und der EG selbst-, ab 31. I 2. 1997 den Export aller gefährlichen Abfälle aus OECD-Ländern in nicht-OECD Länder zu verbieten, auch wenn derartige Verbringungen der Verwertung der Abfälle dienten. Da rechtliche Zweifel bestanden, ob ein solcher Beschluß das Übereinkommen wirksam abändern könne, wurde das Übereinkommen auf der Dritten Konferenz der Vertragsstaaten förmlich geändert. Der Export gefährlicher Abfälle zur Beseitigung von den in einem Anhang VII genannten Vertragsstaaten in andere Vertragsstaaten wurde mit sofortiger Wirkung, der zur Verwertung ab 31. I 2. 1997 verboten; in Anhang VII wurden aufgenommen OECD-Mitglieder, die EG und Liechtenstein.

If Entscheidung 93/98, A Bl.EG 1993, Nr. L 39, S. r; bei dern Bascler Ühercinkommen handelt es sich um ein sog. gemischtes Übereinkommen, das teilweise in den Zuständigkeitsbereich der Mitgliedstaaten, teilweise in den der EG fällt.

I 2 Niche beigetreten sind dem Übereinkommen bisher dic USA, im wesentlichen wohl aus der Befürchtung, es schränke den Handel mit gefährlichen Abfallen zu stark ein. 
Gleichzeitig beschloß die Konferenz, bis zur nächsten Konferenz der Vertragsstaaten eine Liste derjenigen Abfälle auszuarbeiten, die unter das Exportverbot fielen. Diese Liste, bestehend aus einer A- und einer B-Liste, wurde 1997 fertiggestellt und auf der 4. Konferenz der Vertragsstaaten 1998 in Kuching (Malaysia) einstimmig als Anhang VIII und IX in das Übereinkommen aufgenommen.

Am Is. Dezember 1989 schlossen die EG und etwa yo Staaten in Afrika, der Karibik und im Pazifik (AKP-Staaten) in Lomé ein Übereinkommen über die Zusammenarbeit. Das Übereinkommen sah in Artikel 39 ein grundsätzliches Verbor des Handels mit gefährlichen Abfällen zwischen den Vertragsparteien vor. Die EG genehmigte das Übereinkommen $199 \mathrm{r}^{13}$.

\section{OECD-Regelungen}

Die OECD, der sämtliche EG-Mitgliedstaaten sowie andere industrialisierte Staaten - USA, Japan, Kanada, Australien usw. - angehören, hatte an der Ausarbeitung des Baseler Übereinkommens maßgeblich mitgewirkt. Einige Staaten, insbesondere die USA $^{\prime 4}$, waren indessen der Auffassung, das Baseler Übereinkommen enthielte zu weitgehende Beeinträchtigungen des internationalen Handels mit Abfällen. So kam es am 30.3. $1992 \mathrm{zu}$ einem Beschluß des OECD-Rates über die Überwachung der grenzüberschreitenden Verbringung von Abfällen zur Verwertung". Dieser Beschluß galt grundsätzlich nur für Abfallverbringungen zwischen den OECD-Staaten. Er reilte Abfälle in grüne (ungefährliche Abfälle), gelbe und rote (gefährliche Abfälle) Listen ein und legte unterschiedliche Regeln für die Überwachung der Verbringung von Abfällen der verschiedenen Listen fest.

\section{EG-Regelungen für Abfalltransporte}

Die EG hatte bereits 199 die Richtlinien über Abfälle ${ }^{16}$ und gefährliche Abfälle ${ }^{17}$ geändert. Die hier interessierenden Änderungen betrafen

(a) neue Definitionen für »Abfall« und »gefährlicher Abfall«, die nunmehr davon ausgingen, daß gemeinschaftliche Listen klarstellten, was unter Abfällen und gefährlichen Abfällen zu verstehen sei; die EG-Listen wurden 1994 erstellt ${ }^{18}$;

(b) eine ausdrückliche Unterscheidung zwischen der Beseitigung und der Verwertung von Abfällen; die sprachliche Unklarheit der Richtlinie von 1975, daß Beseitigungsvorgänge auch Verwertungsvorgänge einschließen, und die sich auch im Baseler Übereinkommen findet, wurde für die EG damit beseitigt;

(c) die Einführung des Begriffs der »Entsorgungsautarkie« und des Erfordernisses, Abfälle grundsätzlich »in einer der am nächsten gelegenen geeigneten Entsorgungsanlagen « zu beseitigen (Näheprinzip)'`. Beide Grundsätze galten aber ausdrücklich nur für die Beseitigung, nicht aber für die Verwertung von Abfällen.

13 Entscheidung 91/400, ABl.EG 199x, Nr. L 229, S. 1.

14 Wic ausgeführt, haben die USA das Baseler Ubereinkommen zwar unterzeichnet, sind ihm aber nicht beigetreten.

is OECD, Beschluß C (92)93, Environment Monographs No.34, Paris 1992.

I6 Vgl. Richtlinie 9I/rs6, ABl. EG 1991, Nr. L 78, S. 32.

17 Vgl. Richtlinie $91 / 689$, ABI.F.G 1991, Nr. L 377, S. 20

18 Entschcidung 94/3 der Kommission, ABI.EG I 994, Nr. L S, S. I (Abfalle); Entscheidung 94/904 des Rates, AB!.EG 1994, Nr. L 356, S. I4 (gefährliche Abfälle).

r9 Artikel s der Richtlinie 75/442 idF der Richtlinic 91/rs6 (Fn Is). 
Anfang 1993 ersetzte die Gemeinschaft die Richtlinie 84/631 über die Verbringung von gefährlichen Abfällen ${ }^{20}$ durch die Verordnung $259 / 93^{21}$. Die EG-Regelung betraf nunmehr alle, nicht mehr nur gefährliche Abfälle. Für die Unterscheidung zwischen beiden Arten von Abfällen griff die EG nicht auf eigene Kriterien zurück, sondern übernahm das OECD-System der gelben, grünen und roten Listen. Dabei blieben drei Gesichtspunkte unberücksichtigt: zum einen schuf die EG damit ein System, nach dem es eine Liste für gefährliche Abfälle nach der Richtlinie 9I/689 gab konkretisiert durch die Entscheidung $94 / 904^{22}$ - und eine zweite, unterschiedliche Liste für die Verbringung gefährlicher Abfälle. Zum zweiten waren die Listen der OECD erarbeitet worden, um die Verbringung von Abfällen zur Verwertung zu regeln, während die Listen der EG-Verordnung auch für Beseitigungsvorgänge (Deponierung, Verbrennung ohne Energierückgewinnung) galt. Da ein Verwertungsvorgang darauf abzielt, den im Abfall enthaltenen wirtschaftlichen Wert (wieder) nutzbar zu machen, kann ein bestimmter Abfall, der auf der grünen OECD-Liste (nichtgefährlich) steht, dennoch gefährlich sein, z.B. auf Deponien.

Der dritte Gesichtspunkt ist der belangreichste. Die OECD-Listen waren aufgestellt worden, um Verbringungsvorgänge zwischen OECD-Mitgliedern zu regeln; diese Mirglieder sind alle industrialisierten Staaten. Es kann davon ausgegangen werden, daß sie eine ausgeformte Abfallpolitik besitzen, eine Abfallverwaltung, die Abfallverbringungen überwachen und gegebenenfalls unterbinden kann, daß sie Sankrionen aussprechen, kurz: daß sie eine Abfallinfrastruktur besitzen, die bei der Abfallbewirtschaftung einen angemessenen Schutz von Mensch und Umwelt sicherstellt. Für Staaten der Dritten Welt sind diese Voraussetzungen - bei allem Risiko von Verallgemeinerungen - nur selten gegeben.

Das von der Verordnung 259/93 vorgesehene System ist kompliziert. Abfallexporte zur Beseitigung sind verboten ${ }^{23}$. Beim Export von Abfällen zur Verwertung unterscheidet die Verordnung zwischen nichtgefährlichen (grüne Liste, Anhang II) und gefährlichen (gelbe und rote Liste, Anhänge III und IV) Abfällen.

Beim Export von nichtgefährlichen Abfällen in nicht-OECD Länder ${ }^{24}$ hat die EGKommission jedem Staat den Anhang II mit der Liste der nichtgefährlichen Abfälle mitzuteilen und eine schriftliche Nachricht einzuholen, ob und welche Kontrollverfahren für solche Verbringungen gelten sollten. Die Komplexität des Systems wird deutlich, wenn man bedenkt, daß Anhang II etwa I so verschiedene Positionen nichtgefährlicher Abfälle enthält, daß etwa I60 Staaten anzuschreiben sind, daß die Antwort jedes Staates für jede einzelne Position unterschiedlich ausfallen und die möglichen Antworten von "Einfuhr ohne Kontrollen« über "Einfuhr mit Kontrollen entsprechend der gelben Liste akzeptiert", "Einfuhr mit Kontrollen entsprechend der roten Liste akzepticrt« bis zum Unterlassen einer Antwort für einzclne Positionen reichen kann, daß ferner jeder Drittstaat die Möglichkeit hat, - z. B. nach Wahlen, aufgrund von Erfahrungen, nach einem Regierungswechsel oder aus anderen Gründen - seine Meinung jederzeit zu jeder einzelnen Position zu ändern.

Die Europäische Kommission suchte von allen Drittstaaten eine Stellungnahme, ob sie die Einfuhr nichtgefährlicher Abfälle als die Einfuhr von Produkten(!)ansehen

20 Richtlinic $84 / 631$ (Fn 10)

21 Vcrordnung 259/93 zur Uberwachung und Kontrolle der Verbringung von Abfallen in der, in die und aus der Europaischen Gemeinschaft, A.Bl.EG 1993, Nr. L 30, S. I.

22 Entscheidung $94 / 904$ (Fn 18 ).

23 Verordnung 259/93, Artikel 14; eine - hier nicht interessierende - Ausnahme gilt fur Exporte in EFTAStaaten, die auch Vertragsparteien des Baseler Übereinkommens sind.

${ }_{24}$ Für Exporte in OECD-Länder gelten besondere Regeln, Arrikel i7 Abs. 4 und s 
oder, falls sie sie als Abfälle ansahen, welches Kontrollverfahren sie angewandt wissen wollten. Antwortete ein Staat nicht oder erklärte er allgemein, daß er die Einfuhr von nichtgefährlichen Abfällen (grüne Liste) nicht wünschte, dann sollte dies nach Auffassung der Kommission nicht dazu führen, daß Einfuhren in diesen Staat nicht mehr stattfanden; denn diese Länder "sind sich möglicherweise nicht ganz der Bedeutung ihrer Entscheidung für diejenigen ihrer Wirtschaftszweige bewußt, die Abfälle der grünen Liste zur Aufbereitung und Weiterverarbeitung verwenden können « ${ }^{25}$. Die Kommission schlug deswegen eine Verordnung dahingehend vor, daß in solchen Fällen das Kontrollverfahren der roten Liste (gefährliche Abfälle) angewandt werden solle ${ }^{26}$. Da die Kommission die Ausfuhr nichtgefährlicher Abfälle als Handelsregelung, nicht aber als Regelung im Rahmen der Umweltpolitik ansah, stützte sie ihren Vorschlag auf Artikel 113 (Handelspolitik), nicht aber auf Artikel rzos (Umweltschutz).

Über den Vorschlag der Kommission ist noch nicht entschieden. Das Europäische Parlament war der Auffassung, wenn Drittstaaten nicht antworteten oder die Einfuhr nichtgefährlicher Abfälle nicht wünschten, dann müßten ihr fehlendes Interesse oder ihr Wille respektiert werden; Abfälle dürften daher in diese Länder nicht ausgeführt werden ${ }^{27}$. Wie der Rat entscheiden wird, bleibt abzuwarten.

Für gefährliche Abfälle änderte die EG die Verordnung 259/93, um dem Exportverbot des Basler Übereinkommens nachzukommen ${ }^{28-30}$. Die Ausfuhr gefährlicher Abfälle (gelbe und rote Liste) zur Verwertung wurde ab x.x. $x 998$ verboten. $\mathrm{Da}$ im Rahmen des Basler Übereinkommens eine Liste der unter das Ausfuhrverbot fallenden gefährlichen Abfälle noch nicht erstellt war, wurde die Kommission beauftragt, das Ausfuhrverbor so schnell wie möglich an die zukünfrigen Listen des Basler Übereinkommens anzupassen. Gleichzeitig sollten in das Exportverbot die gefährlichen Abfälle der EG-Liste eingefügr werden.

\section{Radioaktive Abfälle}

Für radioaktive Abfälle nahm der Rat 1980 und 1992 zwei Entschließungen für einen Aktionsplan für radioaktive Abfälle an, ging jedoch auf die Ausfuhr derartiger Abfälle aus der Gemeinschaft nicht ein's'. 1994 erließ er eine Richtlinie über die Verbringung radioaktiver Abfälle ${ }^{32}$. Die Richtlinie war der oben erwähnten Richtlinie 84/63I nachgebilder; sie sah eine Genehmigungspflicht für Verbringungen innerhalb der EG vor, bei dem der Antrag im Ausgangsland zu stellen war. Die Behörden des Bestimmungslandes hatten der Verbringung zuzustimmen.

Für die Genehmigung der Ausfuhr aus der Gemeinschaft ${ }^{33}$ in ein Drittland ist eine Zustimmung des Bestimmungslandes jedoch nicht erforderlich. Vielmehr bestimme Artikel $x 2$ insoweit, daß sich die Behörden des Ausgangslandes »mit den Behörden des Bestimmungslandes der Verbringung in Verbindung "setzen. "Sind alle Voraussetzungen für die Verbringung erfüllt« - die Richtlinie enthält keine solchen Voraus-

25 $\mathrm{KOM}(94) 678$ rom 8.2.1995, Rz II

26 Kommission (Fn 25).

27 Europàisches Parlament, Stellungnahme vom 17. 7. 1997, ABI.EG 1997, Nr. C 286, S. 229

28-30 Verordnung $120 / 97$, A Bl.EG 1997, Nr. L 22, S. 14.

3 r Entschlicßungen vom 18. 2. 1980 , ABl.EG 1980, Nr. C 51, S. 1, und vom 15. 6. 1992, ABl.EG r992, Nr. C I 5 , S. 3.

32 Richtlinic 94/3/EURATOM, ABl.EG 1994, Nr. L 35, S. 24

33 Nach Artikel 11 der Richtlinie 94/3 (Fn 32) dürfen Ausfuhren in cinen AKP-Staat und an einen Ort südlich des 60 . Breitengrades niche genchmigt werden. 
setzungen -, so erteilen die Behörden des Ausgangslandes dem Besitzer die Genehmigung zur Verbringung der radioaktiven Abfälle; ssie setzen die Behörden des Bestimmungslandes von der Verbringung in Kenntnis « $^{34}$. Die Verbringung von radioaktiven Abfällen von einem EG-Mitgliedstaat in den anderen ist also an strengere Voraussetzungen geknüpft als die Ausfuhr radioaktiver Abfälle in einen Drittstaat ${ }^{35}$.

\section{Einzelprobleme der Abfallexporte}

Insgesamt sind damit nach knapp zehn Jahren regelnder Maßnahmen die Konturen der Abfallexporte aus der EG in andere(nicht-OECD) Länder festgelegt, auch wenn eine Anzahl Einzelheiten noch zu regeln sind; sie seien hier kurz zusammengefaßt: a. Zu unterscheiden ist zwischen nichtgefährlichen, gefährlichen und nuklearen Abfällen.

b. Nichtgefährliche Abfälle dürfen exportiert werden, wenn sie ausschließlich zur Verwertung bestimmt sind und der importierende Drittstaat seine Zustimmung gegeben hat.

c. Gefährliche Abfälle dürfen nicht exportiert werden, gleichgültig ob es sich um eine Verbringung zur Beseitigung oder zur Verwertung handelt.

d. Der Export von nuklearen Abfällen richtet sich nach der jeweiligen Vereinbarung zwischen dem exportierenden EG-Staat und dem Drittstaat.

Selbstverständlich kann Umweltrecht nicht so einfach sein. Verschiedene Rechtsprobleme stellen diese Grundstruktur in Frage. Im folgenden wird die Problematik der Abfallexporte in Drittstaaten anhand einiger dieser Fragen erläutert.

\section{Die Definition gefäbrlicher Abfälle}

Außerordentlich schwierig ist die rechtliche Definition von "gefährlichen Abfällen«. EG-Recht definierte 1978 als gefährlich solche Abfälle, "die die im Anhang zu dieser Richtlinie aufgeführten Stoffe oder Materialien enthalten oder durch sie verseucht sind, und zwar in einer solchen Beschaffenheit, in solchen Mengen oder Konzentrationen, daß sich daraus eine Gefahr für die Gesundheit oder die Umwelt ergibt « ${ }^{36}$. Diese Definition erwies sich indessen als nicht praktikabel. 1991 ersetzte die EG die Definition durch die Festlegung, daß gefährliche Abfälle solche Abfälle seien, die auf einer Gemeinschaftsliste stünden und die »eine oder mehrere der in Anhang III aufgeführten Eigenschaften aufweisen ${ }^{37}$. Diese Positivliste gefährlicher Abfälle, die ständig fortgeschrieben werden soll, wurdc zwischenzeitlich erstell $t^{3^{8}}$.

34 Das Fehlen eines Zustimmungserfordernisses durch den Bestimmungsstaat widerspricht dem 14. Erwägungsgrund der Richtlinic selbst, der ausführt: ... *muß in Fällen, in denen radioaktive Abfalle in die Gemcinschaft gelangen und/oder sie verlassen, der Drittstaat, der Empfanger ist, und gegebenenfalls der(die) Durchfuhrdrittstaat(en) konsulticrt und unterrichtet werden und müssen ihre Zustimmung gegeben haben $\alpha$.

3s Auf weitere, ähnliche Verschärfungen der Verbringung innerhalb der EG gegenuber Exporten-Dauer der Gultigkeir einer Genehmigung, Fristen für die Information des Bestimmungslandes, Auslegung des Schweigens des Bestimmungslandes, Auflagen des Bestimmungslandes, Mitfuhrungspflicht für Dokumente - wird hier nicht eingegangen.

36 Richtlinie 78/319, Art. I (b); der Anhang führt Arsen, Quecksilber, Cadmium, Blci usw. auf.

37 Richtlinic $9 \mathrm{I} / 689$, Art. 1 Abs. 4 . Anhang III zahlt gefahrenrelevante Eigenschafren der Abfalle auf, etwa ngiftige, $>$ teratogen $\kappa$, pokotoxisch $\propto$ usw.

38 Beschluß 94/904. 
Für den Transport von Abfällen, also auch für den Export, erstellte die EG indessen eine andere Liste gefährlicher Abfälle. Diese Liste ist, wie aufgeführt, mit der Liste des Beschlusses 94/904 nicht identisch.

Das Baseler Übereinkommen über den Transport gefährlicher Abfälle durchlief eine ähnliche Entwicklung. Zunächst wurden als gefährlich solche Abfälle qualifiziert, die zu einer in Anhang I des Übereinkommens aufgeführten Gruppe gehörten, sofern sic eine der im Anhang III aufgeführten Eigenschaften aufwiesen ${ }^{39}$. Mit der Entschejdung 1994/95, den Export gefährlicher Abfälle zu verbieten, wurden genauere Festlegungen notwendig. Demzufolge wurde eine Liste gefährlicher Abfälle erstellt, die unter das Exportverbot fallen. Diese Liste, bestehend aus einer sog. A- und einer BListe, wurde 1998 in das Übereinkommen eingefügt und wird im Herbst 1998 weltweit gültig werden.

Das Problem dieser Liste sei an einem Beispiel dargestellt, das in englischer Sprache wiedergegeben wird, weil es eine offizielle deutschsprachige Liste der Baseler Listen bisher nicht gibt: Nach der Baseler A-Liste sind gefährliche Abfälle »metal wastes and waste consisting of alloys of lead «; doch sind Abfälle, die ausdrücklich in der B-Liste aufgeführt sind, nichtgefährlich (A roro). In der B-Liste findet sich der Eintrag "Clean, uncontaminated metal scrap, including alloys, in bulk finished form (sheet, plate, beams, rods, etc) of ..lead scrap (but ecluding lead-acid batteries" (B rozo). Außerdem findet sich dort der Eintrag "Refractory metals containing residues" (B хозо). Schließlich enthält die A-Liste den allgemeinen Eingangssatz, daß der Einschluß von Abfällen in die Liste A den Rückgriff auf Anhang III des Übereinkommens (gefährliche Eigenschaften) nicht ausschließe, um darzulegen, daß ein Abfall nicht gefährlich ist ${ }^{\circ}$.

Man mag sich vorstellen, wie in einem konkreten Fall die Einfuhrbehörden eines Drittstaates anhand dieser Listen prüfen, ob eine bestimmte Containerladung von Abfällen nun in die Liste A odcr die Liste B fällt. Meinungsverschiedenheiten, Mißverständnisse und Umgehungen der Regelungen scheinen vorprogrammiert.

Insgesamt hat bei der Ausarbeitung der Liste nach dem Baseler Übereinkommen wohl eher die Überlegung im Vordergrund gestanden, welche Abfallexporte gestattet bleiben und welche verboten sein sollten. In anderen Worten: die Listen gingen nicht so sehr von einer Gefährlichkeit der Abfälle als vielmehr von dem Wunsch oder der Notwendigkeit aus, Exporte dieser Abfälle in Drittländer (weiter) zuzulassen.

Damit gehen die beiden Grundgedanken des Exportvcrbots für gefährliche Abfälle, die den Entscheidungen im Rahmen des Baseler Übereinkommens zugrundelagen, verloren: zum einen erklärten zahlreiche nicht-OECD-Staaten selbst, sie seien zu einer umweltgercchten Behandlung, Verwertung, Lagerung und Beseitigung gefährlicher Abfälle nicht in der Lage, weil es an der notwenigen Infrastruktur für ein derartiges Abfall-Management - Gesetze, Verwaltungsapparac, Kontrollmechanismen, Untersuchungsämter, Sanktionsmöglichkeiten usw. - fehle. Zum anderen lag dem Verbot der Gedanke zugrunde, daß ein Industriestaat seine gefährlichen Abfälle auch selbst entsorgen, sie aber nicht - auch nicht unter dem Etikett einer Verwertungin nicht-OECD-Staaten exportieren sollc.

Man wird gcspannt sein dürfen, ob die Baseler Listen klarstellen helfen, welche Abfallexporte verboten sind und welche nicht. Die EG wird ihr Recht nunmehr den internationalen Regeln anpassen müssen. Dabei steht sie vor der Frage, ob die strengeren gemeinschaftlichen Listen auch für Exporte in Drittländer gelten sollen,

39 Bascler Übercinkommen, Art. 1 (a).

40 Auch die B-Liste enthalt den Eingangssatz, daß im Einzelfail ein dargelegt werden konne, daß auf der BListe aufgefuhrter Abfalistoff gefahrlich sei. 
oder ob die EG ihr Schutzniveau auf das vom Baseler Übereinkommen herabsenken soll. Politisch spricht einiges dafür, eine weltweite Liste gefährlicher Abfälle festzulegen, deren Export in nicht-OECD-Länder verboten ist.

Andererseits ist eindeutig, daß die Ausarbeitung von Listen gefährlicher Abfälle nach dem Baseler Übereinkommen, an der mehr als hundert Länder beteiligt sind, weniger Abfälle erfaßt als die EG-Listen. Das Baseler Übereinkommen sieht insoweit in Artikel ${ }_{4}$ Abs. I I ausdrücklich die Möglichkeit für einzelne Vertragsparteien vor, strengere Vorschriften zum Schutz der Umwelt zu erlassen. Es macht auch wenig Sinn, gemeinschaftlich als umweltpolitisch notwendig erkannte und einstimmig festgelegte Standards aufgrund internationaler Entwicklungen wieder abzubauen.

Die EG orientiert sich offenbar dahin, daß diejenigen Abfälle, die im Rahmen des Baseler Übereinkommens als nichtgefährlich (Liste B) eingestuft wurden, dem Exportverbot auch dann nicht unterliegen, wenn diese Abfälle in der EG selbst, in der Liste der gefährlichen Abfälle ${ }^{41}$ oder in der Verordnung über den Transport von Abfällen ${ }^{42}$, als gefährlich eingestuft sind. Diese Regelung, die von allen Mitgliedstaaten befürwortet wird, orientiert den Umweltschutz an den weltweiten Standards des Baseler Übereinkommens, nicht an den bisherigen, strengeren Vorschriften des Gemeinschaftsrechts. Mit einer endgültigen Entscheidung ist noch 1998 zu rechnen.

\section{Der Begriff des Industriestaates}

Die Entscheidung 1994 des Baseler Übereinkommens sah ein Exportverbot für gefährliche Abfälle von OECD- in nicht-OECD Staaten vor. Exporte von OECDStaaten in andere OECD-Staaten oder von nicht-OECD-Staaten in andere nichtOECD Staaten sollten dagegen möglich bleiben. Als diese Entscheidung 1995 in das Baseler Übereinkommen aufgenommen wurcle, wurde das Verbot erweiternd dahingehend formuliert, daß Exporte von Mitgliedern der OECD, der EG und von Liechtenstein in andere, nicht zu dieser Gruppe gehörenden Länder verboten sein sollten; Liechtensteins Aufnahme in die Liste der industrialisierten Länder war eher ein technisch-administratives Mißverständnis als eine wohlüberlegte und-diskutierte Entscheidung. Die EG übernahm denn auch in ihrer Entscheidung, die Exporte gefährlicher Abfälle zur Verwertung ab I. I. 1998 zu verbieten, Liechtenstein nicht auf, so daß Exporte gefährlicher Abfälle auch nach Liechtenstein ab I. I. 1998 verboten sind.

Um nicht von Abfallexporten seitens der EG oderer anderer OECD Mitgliedstaaten abgeschnitten zu werden, wohl auch aus Prestige- und anderen Gründen, versuchten eine Reihe von Schwellenländern, in die Liste der industrialisierten Länder aufgenommen zu werden, selbst um den Preis, daß sie damit Exporte in nicht-OECD Staaten nicht mehr durchführen können. 1998 lagen der Konferenz des Baseler Übereinkommens Anträge von Israel, Slowenien und Monaco vor, in die Liste industrialisierter Länder aufgenommen zu werden. Nach langen, lebhaften Diskussionen lehnte die Konferenz diese Anträge ab, solange das Exportverbot nicht weltweit in Kraft seids.

Trotz dieser Entscheidung ist zu erwarten, daß in den kommenden Jahren neue Anträge gestellt werden, in die Liste der »Industrieländer « aufgenommen zu werden. Auch

41 Entschcidung $94 / 904$ (Fn r 8).

42 Verordnung $259 / 93$ ( $\mathrm{F}_{\mathrm{n}} 2 \mathrm{r}$ ).

43 Bis März $x 998$ haben nur eine geringe Zahl von Staaten das Exportverbot des Baseler Ubereinkommens ratifiziert; hierzu gehoren insbesondere die EG, mehrere ihrer Mitgliedstaaten und Norwegen. 
neue Beitritte zur $\mathrm{OECD}$ oder zur EG würden die Liste verlängern. Wieweit also der Grundsatz, daß Abfallexporte von Industrieländern in die Dritte Welt nicht mehr stattfinden sollen, auf die Dauer aufrechterhalten bleibt, wird abzuwarten sein.

Der Gedanke, Abfallexporte aus OECD-Ländern in die Dritte Welt zu verbieten, erschien in der Vergangenheit geeignet, den Begriff »Industrieland « einigermaßen auszufüllen. Da die OECD eine Reihe von Grundregeln für die Abfallbewirtschaftung in ihren Mitgliedsländern festgelegt hatte, konnte, so der Grundgedanke des Verbots nach dem Baseler Übereinkommen, davon ausgegangen werden, daß die OECD-Mitgliedstaaten Anlagen und Mechanismen besaßen, um die Behandlung und Beseitigung gefährlicher Abfälle umweltgerecht durchzuführen. Ob dies tatsächlich immer der Fall ist, mag wohl bezweifelt werden.

Dann aber stellt sich die Frage, wie »Industrieland « anders definiert werden sollte. Das Baseler Übereinkommen spricht insoweit allein von der Nowendigkeit einer »umweltgerechten" Behandlung oder Beseitigung, ohne den Begriff zu definieren oder näher zu beschreiben. Jeder Staat könnte daher geltendmachen, er entsorge seine Abfälle »umweltgerecht $\propto$. Für ein ordnungsgemäßes Funktionieren des Baseler Übereinkommens wird daher fast alles von Kontrollen abhängen, durch die nachgeprüft wird, ob eine umweltgerechte Entsorgung stattfindet. Aber auf internationaler Ebene wird sich kein Drittstaat - etwa Israel oder Slowenien, aber auch Indien, Brasilien oder die Philippinen - daraufhin kontrollieren lassen, ob die umweltgerechte Bebandlung oder Beseitigung gefährlicher Abfälle in seinem Land auch tatsächlich gewährleistet ist; derartige Kontrollen wurden stets mit dem Grundsatz der uneingeschränkten staatlichen Souveränität abgelehn $\mathrm{r}^{+4}$. Damit droht der Kreis der Industrieländer aufgrund einseitiger, nicht nachprüfbarer Erklärungen einzelner Staaten erweitert zu werden, was mittelfristig das gesamte Exportverbot in Frage stellt.

\section{Zweiseitige Abreden}

Das Verbot, Abfälle in nicht-OECD Staaten auszuführen, wurde im Baseler Übereinkommen in einem neuen Arrikel 4a verankert. Damit stellt sich die Frage, wie sich diese Vorschrift zu Artikel 1 I des Baseler Übereinkommens verhält ${ }^{\text {t5}}$. Kann also das Exportverbot für gefährliche Abfälle durch bilaterale Absprachen umgangen werden? Der Wortlaut von Artikel 1 I, der den neuen Artikel 4a ja gerade nicht erwähnt, scheint eindeutig gegen eine solche Möglichkeit zu sprechen. Zudem bezieht sich Artikel 4 Abs. s nur auf Vereinbarungen mit Nichtvertragsstaaten, für die Artikel I I eine Ausnahmeregelung vorsieht.

Trotz dieser klaren Rechtslage haben einige Staaten - Australien, Neuseeland, wohl auch Kanada - die Auffassung geäußert, Abweichungen von dem Exportverbor könnten nach Artikel Ir vereinbart werden. Zur Begründung führten sie an, die Nichterwähnung von Artikel 4a in Artikel II sei unschädlich, weil Artikel 4a ja erst

44 Innerhalb der OECD und der EG bestehen bestimmte Kontrollmechanismen.

4 Artike! ru lautet: Unbeschadet des Arcikels 4 Absatz s können die Vertragsparteien zweiseitige, mehrseitige und regionale Ubereinkünfte oder andere Vereinbarungen über dic grenzüberschreitende Verbringung gefàhrlicher Abfälle oder anderer Abfälle mir Vertragsparteien oder Nichtvertragsparteien schlieBen .... Die Vertragsparteien notifizieren dem Sekretariat ... alle Übcreinkünfte und anderen Vercinbarungen, die sie, bevor dieses Ubereinkonmen fur sic in Kraft trat, zum Zwecke der Kontrolle der... Verbringung gefährlicher Abfälle und anderer Abfallc geschlossen haben. Das Übereinkommen berührt nicht die aufgrund solcher Übercinkünfte erfolgende grenzüberschreitende Verbringung. *

Arcikel 4 Abs. 5 des Ubereinkommens lautec:n Die Vertragsparteien erlauben weder die Ausfuhr gefährlieher Abfälle oder anderer Abfälle in eine Nichtvertragspartei noch deren Einfuhr aus einer Nichtvertragsparteik. 
später in das Übereinkommen eingefügt worden sei. Jedenfalls seien bilaterale Abkommen gültig, die vor Inkrafttreten des Exportverbots geschlossen worden seien.

Da das Exportverbot völkerrechtlich bisher nicht in $\mathrm{Kraft}$ getreten ist, kommt diesen Argumenten erhebliches politisches Gewicht zu, auch weil nicht klar ist, wie der Abschluß und die Durchführung derartiger bilateraler Übereinkommen verhindert werden könnten. Auf internationaler Ebene gibt es nun einmal keine Sanktionsmöglichkeiten. Nach dieser Auffassung könnte also jeder Vertragsstaat gegenwärtig bilaterale Abkommen nach Artikel II des Übereinkommens schließen und sie dann nach Inkrafttreten des Exportsverbots durchführen.

Die EG hat von der Möglichkeic, bilateralc Abkommen nach Artikel Ir zu schließen, keinen Gebrauch gemacht, und plant solche Abkommen auch nicht. Das 1997 beschlossene Exportverbot gilt also uneingeschränkt. Erst die Zukunft wird zeigen, ob es bei diesem Zustand bleibt. Denn wenn etwa wegen des EG-Exportwerbots bestimmte Ströme für wiederverwertbare gefährliche Abfälle unterbrochen werden und dann andere Staaten, etwa Australien, in die so entstandenen Lücken stoßen, mag der Druck auch innerhalb der EG steigen, nach Möglichkeiten für eine flexiblere Ausgestaltung des Exportverbots zu suchen.

\section{Illegale Verbringungen}

Sowohl nach dem Baseler Übereinkommen (Artikel 9) als auch nach der Verordnung $259 / 93$ der EG (Artikel 26) ist ein illegales Verbringen von Abfällen unzulässig. Beide Texte zählen Fälle auf, die als illegale Verbringung gelten. Illegal ist eine Verbringung insbesondere dann, wenn die erforderlichen Vorausinformationen nicht übermittelt werden, wenn die erforderliche Zustimmung des Einfuhrstaates nicht vorliegt oder wenn die Begleitpapiere nicht mit den tatsächlichen Daten übereinstimmen. Die Abfälle sind sodann vom Exporteur oder dem Ausfuhrstaat zurückzutransportieren, sofern die Verantwortung für die illegale Verbringung im Verantwortungsbereich des Exporteurs liegt; liegt die Verantwortung beim Importeur, so hat der Einfuhrstaat die Entsorgung der Abfälle sicherzustellen.

Diese nur in Umrissen dargestellte Regelung kann selbstverständlich die illegale Verbringung von Abfällen in die Dritte Welt nicht unterbinden. Da in Ländern der Dritten Welt - und man sollte getrost eine Reihe von Ländern aus dem ehemaligen Herrschafts- oder Einflußbereich der Sowjet-Union hierher rechnen - nicht selten Infrastrukturen für eine sachgerechte Abfallbewirtschaftung wie gesetzliche Vorschriften, Genehmigungs- und Überwachungsbehörden, Polizeistrukturen, Verarbeitungs- und Beseitigungsanlagen, Untersuchungsämter usw. fehlen, die Umgehung bestehender Vorschriften leicht, die Unbestechlichkeit von Behörden nicht stets gesichert und das allgemeine Lebensniveau niedriger ist als in den Industrieländern, lohnt es sich oft, insbesondere gefährliche Abfälle, deren Entsorgung in der Gemeinschaft kostenaufwendig ist, zu wesentlich geringeren Kosten in Drittländern zu entsorgen. Jedenfalls teilweise benutzt die organisierte Kriminalität den illegalen Export von Abfällen als Geldquelle ${ }^{4}$. Naturgemäß gibt es keinerlei statistische Daten über den Umfang illegaler Abfallverbringungen.

Die häufigste Form der illegalen Abfallverbringung ist das Fälschen der Begleitpapiere oder das Nichtübereinstimmen von Begleitpapieren mit dem tatsächlichen

46 Vgl. z. B. S. Bugdahn, Abfallkriminalität als Problem der deutschen und europäischen Rechtspolitik. Munster 1997; Legambiente (Hrsg.) Le nuove fronitere dell' ecomafia. Ms., Rom 1998. 
Inhalt der Abfälle. Häufig ist das Undeklarieren der Begleitpapiere von gefährlichen in nichtgefährliche Abfälle, das Mischen gefährlicher mit nichtgefährlichen Abfällen oder das Exportieren von im Inland nicht mehr marktfähigen Produkten; diese letzteren werden nicht als "Abfälle" exportiert, sondern als "gebrauchte Güter «, so daß auf sie die gesamte Abfallgesetzgebung nicht mehr anwendbar ist ${ }^{47}$.

Die Entsorgung illegal verbrachter Abfälle gestaltet sich regelmäßig schwierig, weil wegen der anfallenden Kosten etwaige Meinungsverschiedenheiten über Herkunft, Zusammensetzung und Verantwortung für die Abfälle Gewicht bekommen. Staaten scheuen nicht selten die Übernahme der Entsorgung, weil sie hierin ein Eingeständnis zu sehen scheinen, daß bei der Ausfuhr nicht alles ordnungsgemäß abgelaufen sei. Das Bekanntmachen der illegale Verbringung durch Presse, Funk und Fernsehen oder das Einschalten der internationalen oder gemeinschaftlichen Institutionen scheint das Lösen derartiger Konflikte zu beschleunigen.

Um die etwaige Rückführung von Abfällen zu erleichtern, sieht die EG-Verordnung vor, daß für jede Verbringung von Abfällen eine Sicherheitsleistung oder der Nachweis einer entsprechenden Versicherung erforderlich ist. Natürlich hilft diese Sicherheitsleistung nur in solchen Fällen, in denen eine ursprünglich ordnungsgemäße Verbringung nachträglich rückabzuwickeln ist; bei kriminellen Aktivitäten hilft sie nicht.

\section{Sanktionen}

Das Baseler Übereinkommen erklärt, die Vertragsparteien seien »der Auffassung, daß der unerlaubte Verkehr mit gefährlichen Abfällen oder anderen Abfällen eine Straftat darstellt", überläßt es aber den Vertragsparteien, Sanktionen für illegale Verbringungen festzulegen. Die EG-Verbringungsverordnung sieht lapidar vor: "Die Mitgliedstaaten verbieten und ahnden die illegale Beförderung durch geeignete rechtliche Maßnahmen«. Irgendwelche zuverlässigen Angaben über die festgelegten sowie die Zahl der wegen illegaler Verbringung ausgesprochenen Sanktionen gibt es nicht. Allgemein scheint der illegale Export von Abfällen in die Dritte Welt eher als Kavaliersdelikt denn als ernsthafte Straftat angesehen zu werden.

Über den Export nukJearer Abfälle von Industriestaaten in Nichtindustriestaaten ist wenig Verläßliches bekannt. Es hat den Anschein, als werde ein Exportverbot für radioaktive Abfälle in jene Länder nicht angestrebt. Die Europäische Gemeinschaft hat den Export radioaktiver Abfälle in Staaten des afrikanischen, karibischen und pazifischen Raums 1989 verboten. Im übrigen gibt es auf weltweiter Ebene seit 1990 einen nichtverbindlichen Kodex der Internationalen Atomenergie-Kommission, der jedem Staat das souveräne Recht einräumt (!), das Verbringen radioaktiver Abfälle auf seinem Gebiet zu verbieten. Der Kodex sieht ferner ein System der Vorabinformation und Zustimmung der von der Verbringung berührten Staaten ("prior informed consent $\ll)$ vor ${ }^{8}$.

47 Rechtlich ist ein Gegenstand, dessen sich der Inhaber entledigen muß, weil er den für die Marktfähigkeit geltenden Regeln nicht mehr entsprichr, als Abfall cinzustufen; im Export läßt sich die Unterscheidung zwischen reparaturfäligen gubrauchten Gütern und Abfällen indessen besonders leiche verwischen, weil im Drittland andere Vorschriften und andere Kosten für die Reparatur von Gebrauchsgritern Anwendung finden.

$4^{8} \mathrm{Vg}$ !. EG-Kommission, Mirtcilung über die gegenwärtige Lage und die Aussichten für die Bewirtschaftung radioaktiver Abfälle in der Europäischen Gemeinschaft, KOM (93) 88 vom r.4. 1993. 
Als Resumé läßt sich feststellen, daß die rechtliche Entwicklung des EG-Umweltrechts und des Baseler Übereinkommens über die Verbringung gefährlicher Abfälle in den letzten zehn Jahren dazu geführt haben, daß der Export gefährlicher Abfälle von Industriestaaten in Nichtindustriestaaten schrittweise illegal wird, obwohl in vielen Details weitere Regelungen erforderlich bleiben, um diesem Grundsatz Geltung zu verschaffen. Die Rechtslage ist weniger klar bei nuklearen Abfällen, bei denen es ein durch internationales Übereinkommen oder EG-Regelung festgelegtes grundsätzliches Exportverbot nicht gibt. Nichtgefährliche Abfälle, die der Verwertung dienen, dürfen mit Zustimmung des Drittstaats exportiert werden.

Das Interesse der industrialisierten Staaten kann also leicht in die Richtung gehen, möglichst viele Abfälle als nichtgefährlich zu erklären, um sie dem Exportverbot zu entziehen. Das Interesse krimineller Akteure wird dahin gehen, gefährliche Abfälle mit nichtgefährlichen zu mischen und das Ergebnis als nichtgefährliche Abfälle zu deklarieren, gefährliche Abfälle falsch, nämlich als nichtgefährliche Abfälle zu deklarieren oder gefährliche Abfälle mit »Produkten« - Sägespäne, Kohle usw. - zu mischen und das Mischprodukt als Produkt auszugeben.

Der Gesetzgeber wird damit als vordringliche Aufgabe klare Regelungen dahingehend treffen müssen, was ein gefährlicher Abfall ist. Ob die EG in Zukunft ihre gegenwärtige Unterscheidung aufrechterhalten kann, man müsse zwischen »gefährlichen Abfällen für die Genehmigung von Anlagen, die Lagerung und Entsorgung* und "gefährlichen Abfällen für den Transport" unterscheiden, ist zweifelhaft. Denn diese Unterscheidung läuft darauf hinaus, innerhalb der EG bestimmte Abfälle als gefährlich zu erklären, die nicht dem 1997 beschlossenen Verbot des Exports gefährlicher Abfälle unterliegen. Nur allzu leicht kann dann der politische Vorwurf erhoben werden, die EG messe mit zweierlei Maß, indem sie Abfälle, die sie hinsichtlich des Verkehrs innerhalb der Gemeinschaft als gefährlich einstuft, hinsichtlich des Exports in nicht-OECD-Staaten als nichtgefährlich einstuft. Genau hierauf läuft allem Anschein nach die Politik der EG hinaus.

Wie in fast allen Bereichen des Umweltrechts zeigt sich hinsichtlich der übrigen Aspekte auch für die Verbringung von Abfällen, daß der Durchsetzung und praktischen Anwendung der Umweltregelungen das entscheidende Gewicht zukommt. Allein gesetzliche Regelungen können betrügerische oder mafiotische Machenschaften bei der Verbringung von Abfällen nicht verhindern oder beenden. Entscheidend wird die Entschlossenheit sein, Umweltkriminalität mit ausreichenden polizeilichen und administrativen Mitteln zu bekämpfen, Nachlässigkeiten, Bestechlichkeiten und Unterlassungen nicht hinzunehmen, Fehlverhalten aufzudecken, Sanktionen auch auszusprechen und endlich auch Verantwortung dafür zu übernehmen, daß die Umwelt in der Dritten Welt - besser: außerhalb der Industrieländer - nicht durch Produkte, Produktionsweisen, Abfälle oder andere Ursachen aus den Industrieländern beeinträchtigt wird. 